\title{
Influence of Laboratory Generated Turbulence on Phase Fluctuations of a Laser Beam
}

\author{
M. Carnevale, B. Crosignani, and P. Di Porto
}

\begin{abstract}
An experiment is performed the aim of which is to investigate the phase fluctuations of a laser beam induced by artificially generated thermal turbulence. This is achieved by observing the displacements of a fringe pattern obtained by means of a Mach-Zehnder interferometer. The temporal decay of the mean square refractive index fluctuation is studied. An interpretation of the results is given on the basis of the theory of an isotropic turbulent scalar field.
\end{abstract}

\section{Introduction}

The atmosphere undergoes random variations of its density and temperature that result in temporal and spatial fluctuations $\Delta n(\mathbf{r}, t)$ of its refractive index $n(r, t)$. Generally, these are described in terms of a statistical interpretation, based on the introduction of the correlation function defined through the ensemble average $\left\langle\Delta n(\mathbf{r}, t) \Delta n\left(\mathbf{r}^{\prime}, t^{\prime}\right)\right\rangle$. Its form is usually specified in a way, based on the Kolmogoroff theory of locally isotropic turbulence, that permits a first approach to the problem of wave propagation in the atmosphere. ${ }^{1,2}$

As is well known, atmospheric turbulence is responsible for fluctuations in the parameters that characterize an electromagnetic beam, that is, its amplitude, frequency, and phase. These changes can be observed by means of a number of effects, such as diffraction, scintillation, and line broadening at the receiving optical system. So far, this argument has been faced, both theoretically ${ }^{3}$ and experimentally ${ }^{4}$ by many authors. A great amount of work has been devoted to the study of the behavior of an em beam over a long path outdoors, because of the importance of turbulence with respect to communications. On the other hand, the investigation of laboratory generated turbulence allows, in some sense, a more careful insight into the structure of refractive index fluctuations, owing to more effectively controlled experimental conditions. This kind of experiment, beside the possibility of checking turbulence theory, may furnish some useful model for the free atmosphere.

We wish here to report an experiment, the aim of which is to measure phase fluctuations of a laser beam induced by artificially generated turbulence. This is done by observing the displacements of a fringe pat-

The authors are with the Fondazione U. Bordoni, Istituto Superiore Poste e Telecomunicazioni, Roma, Italy.

Received 27 November 1967. tern, which is obtained by means of a Mach-Zehnder interferometer. We generate refractive index fluctuations on a given plane by using a hot wire grid. The turbulence region is then shifted by means of a suitable stream along the positive $x$ direction (see Fig. 1), so that it is possible to study the temporal decay of the mean square refractive index fluctuation as a spatial decay. This is achieved by examining the mean square fluctuations of the fringes as a function of the distance of the traveling beams from the hot wire. A theoretical interpretation of the results can be given on the basis of the theory of an isotropic turbulent scalar field.

\section{Phase Fluctuations in an Isotropic Turbulent Scalar Field}

We wish here to evaluate the phase variations of a laser beam through a simple application of the theory of isotropic and homogeneous turbulence. ${ }^{5}$ Given a scalar quantity $\Gamma=\bar{\Gamma}+\gamma$, the simplest way of describing its behavior in a turbulent field is based on the introduction of the correlation between the fluctuations about the average value, which is defined through the ensemble average:

$$
\left[Q_{\gamma \gamma}(r, t)\right]_{A, B}=\left\langle\gamma_{A} \gamma_{B}\right\rangle .
$$

Here, $\gamma_{A}$ and $\gamma_{B}$ denote the value of $\gamma$ at the points $A$ and $B$ located at a distance $r$ from each other; the vector symbol is omitted because of isotropy.

Under general hypothesis, it can be shown that the following diffusion equation holds:

$$
\frac{\partial}{\partial t} Q_{\gamma \gamma}(r, t)=2 \chi \frac{1}{r^{2}} \frac{\partial}{\partial r}\left[r^{2} \frac{\partial}{\partial r} Q_{\gamma \gamma}(r, t)\right] .
$$

If one assumes small values of the Reynolds number of turbulence (see Ref. 5, p. 229), no interaction term between $\gamma$ and the velocity field are present. We are interested in thermal fluctuations, in which case $\Gamma=$ $C_{p} T$ is the amount of heat per unit of mass and $\chi$ in Eq. (2) specifies the heat conductivity of the fluid. 


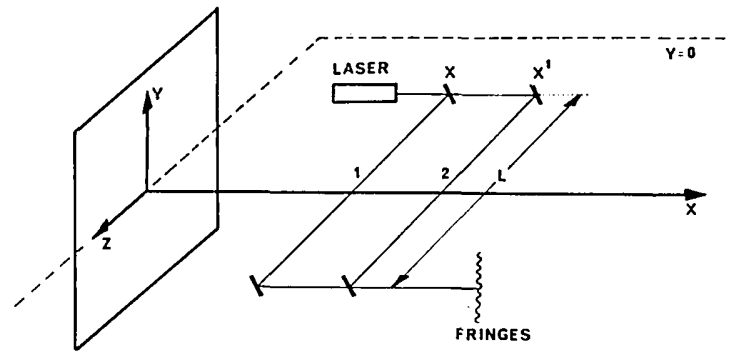

Fig. 1. Geometry of the problem.

The effect of thermal turbulence on the refractive index of the medium can be evaluated through the relation:

$$
\Delta n=-[(\bar{n}-1) / \bar{T}] \Delta T,
$$

which is valid under isobaric conditions. A suitable solution for Eq. (2) reads (see Ref. 5, p. 230):

$$
\left.Q_{\gamma \gamma}(r, t)=\left(C / t^{3 / 2}\right) \exp -r^{2} / 8 \chi t\right) .
$$

Through Eqs. (3) and (4), one is now able to evaluate the correlation function of refractive index. In our classical Mach-Zehnder experiment, in which the initial beam is split in two parallel beams that are made to interfere after traveling through the medium (see Fig. 1), the characteristic quantity describing the stochastic variations of the position of the fringes obtained on a screen is the mean square value of the phase fluctuations:

$$
\begin{aligned}
\left\langle(\Delta \phi)^{2}\right\rangle= & \left\langle\left\{k_{0} \int_{0}^{L}[\Delta n(1)-\Delta n(2)] d z\right\}^{2}\right\rangle \\
& =k_{0}{ }^{2} \int_{0}^{L} d z \int_{0}^{L} d z^{\prime}\left[\left\langle\Delta n(x, 0, z) \Delta n\left(x, 0, z^{\prime}\right)\right\rangle\right. \\
& \left.+\left\langle\Delta n\left(x^{\prime}, 0, z\right) \Delta n\left(x^{\prime}, 0, z^{\prime}\right)\right\rangle-2\left\langle\Delta n(x, 0, z) \Delta n\left(x^{\prime}, 0, z^{\prime}\right)\right\rangle\right] .
\end{aligned}
$$

Here, $L$ is the common length of the two beams, which are supposed to lie in the plane $y=0$, and $k_{0}$ represents the wavenumber of the incident light.

Thermal fluctuations are generated in a region around the plane $x=0$, and then are made to stream along the positive $x$ direction by means of a soft wind of given velocity $v$. If the distance between the two beams is small compared with their distance from the plane $x=$ 0 , the described geometry implies that Eq. (4) has to be evaluated at the time $\bar{\tau} \sim x / v$, in order to get $\left\langle(\Delta \phi)^{2}\right\rangle$ from Eq. (5). By means of Eqs. (3), (4), and (5) one obtains:

$$
\begin{aligned}
\left\langle(\Delta \phi)^{2}\right\rangle= & \frac{4 C k_{0}{ }^{2}(\bar{n}-1)^{2}}{C_{p}{ }^{2} \bar{T}^{2}} \frac{1}{t^{3 / 2}} \int_{0}^{L} d z \int_{0}^{z} d s \exp \\
& \quad\left(-s^{2} / 8 \chi \bar{l}\right)\left\{1-\exp \left[-\left(x-x^{\prime}\right)^{2} / 8 \chi \bar{l}\right]\right\} \quad(6) \\
= & D \frac{1}{i}\left\{1-\exp \left[-\left(x-x^{\prime}\right)^{2} / 8 \chi \bar{t}\right]\right\} \int_{0}^{L} d z \operatorname{Erf}\left[z /(8 \chi \bar{l})^{\frac{1}{2}}\right],
\end{aligned}
$$

with $s=\left|z-z^{\prime}\right|$ and $D=2 C k_{0}{ }^{2}(\bar{n}-1)^{2}(8 \pi \chi)^{\frac{1}{2}} / C_{p}{ }^{2} \bar{T}^{2}$, having introduced the error function:

$$
\operatorname{Erf}(z)=\frac{2}{(\pi)^{\frac{1}{2}}} \int_{0}^{z} \exp \left(-x^{2}\right) d x
$$

In our experimental conditions, $\exp \left[-\left(x-x^{\prime}\right)^{2} / 8 \chi \bar{t}\right]$ is negligible with respect to unity, so that

$$
\begin{aligned}
\left\langle(\Delta \phi)^{2}\right\rangle= & \frac{D}{\bar{t}}(8 \chi \bar{t})^{\frac{1}{2}} \int_{0}^{L /(8 \chi \bar{t})} \operatorname{Erf}(\xi) d \xi \\
= & \frac{D}{\bar{t}}(8 \chi \bar{t})^{\frac{1}{2}}\left[\frac{L}{(8 \chi \bar{t})^{\frac{1}{2}}} \operatorname{Erf}\left(\frac{L}{(8 \chi \bar{t})^{\frac{1}{2}}}\right)\right. \\
& \left.\quad+\frac{1}{(\pi)^{\frac{1}{2}}} \exp \left(-L^{2} / 8 \chi \bar{t}\right)-\frac{1}{(\pi)^{\frac{1}{2}}}\right],
\end{aligned}
$$

which for all significant values of $L$ and $\bar{t}$ can be well approximated by

$$
\left\langle(\Delta \phi)^{2}\right\rangle=D L / \bar{t} .
$$

Remembering now the expression for $\bar{t}$, one also gets

$$
\left\langle(\Delta \phi)^{2}\right\rangle=(D L / x) v .
$$

\section{Experimental Setup and Results}

Equation (8) has been tested as a function of the average time $\bar{t}$, in which the turbulent zone is shifted from the plane $x=0$ to the interfering beams.

The turbulent region is produced inside a tube about $30-\mathrm{cm}$ in diameter by a grid of heated nichrome wires that generates thermal fluctuations of the order of $1^{\circ}$ (see Fig. 2). The turbulent region is transported by an air drift produced by a blower placed at the extremity of the tube opposite to the grid; the range of air velocities lies between $10 \mathrm{~cm} / \mathrm{sec}$ and $30 \mathrm{~cm} / \mathrm{sec}$. Two parallel beams are obtained from a $6328-\AA$ laser source by means of a Mach-Zehnder interferometer, their distance being $10 \mathrm{~cm}$. The two beams travel through the medium for a length $L=$ constant and are afterwards made to interfere. The average separation between the grid and the beams is varied between 1 and $3 \mathrm{~m}$, thus exploring the time range between 4 sec and 30 sec.

The interference pattern strikes the oscillating mirror of a galvanometer from which it reflects to the detector slit. A photomultiplier is placed behind the slit. The mirror is made to oscillate around an axis parallel to the fringes in such a way as to obtain the scanning of the

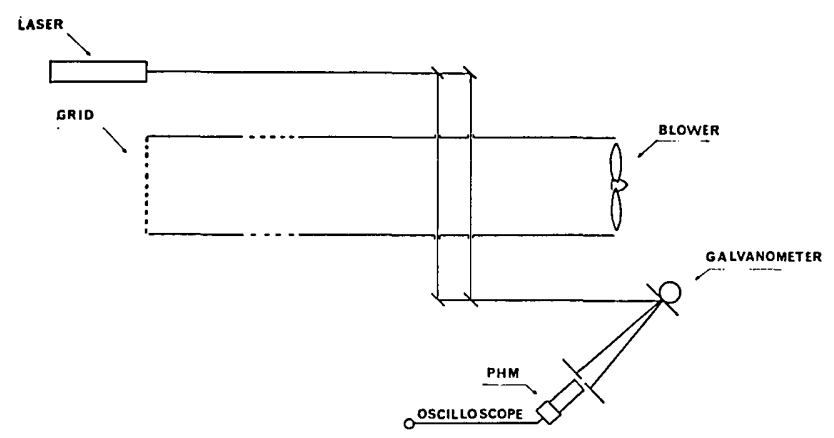

Fig. 2. Experimental setup. 


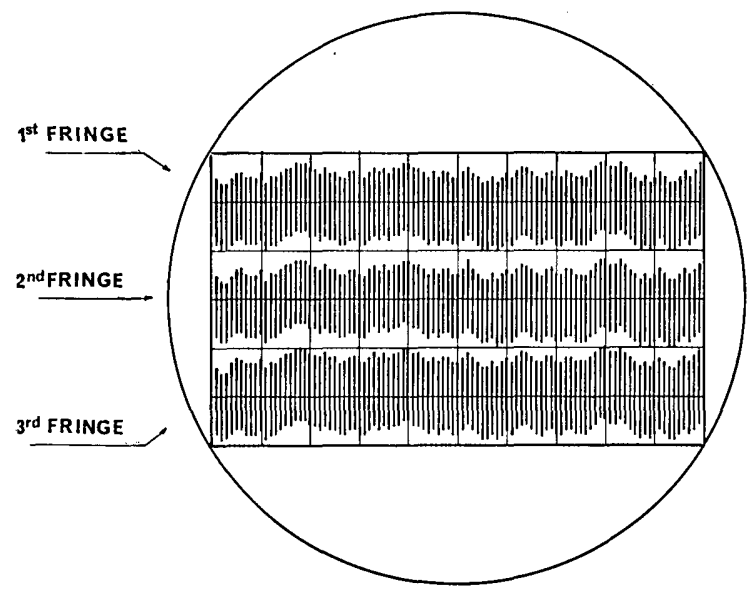

Fig. 3. Schematic plot of the oscilloscope pattern.

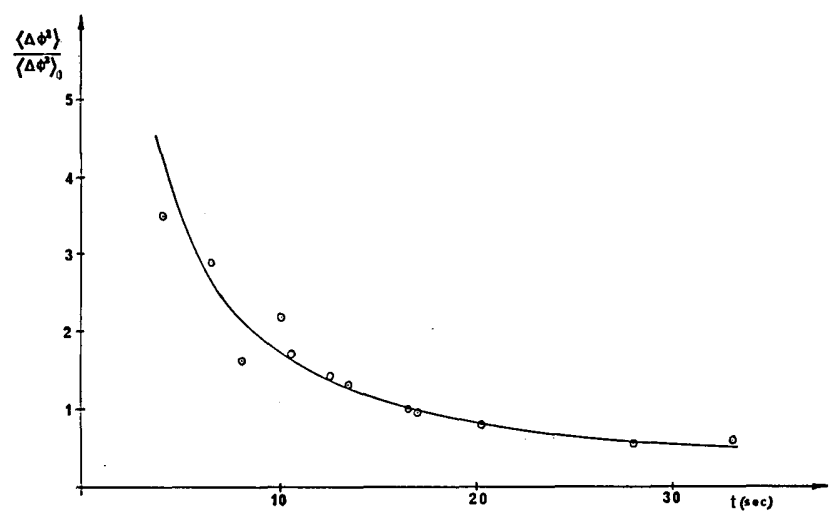

Fig. 4. Mean square value of the phase fluctuations as a function of time.

interference pattern on the slit. This oscillation is obtained by sending to the galvanometer coil a current of triangular shape; the same signal is sent to the vertical axis of an oscilloscope. A slow time scan on the horizontal axis thus gives a raster of vertical lines. In order to record the fringe positions and their displacements, the photomultiplier signal is sent to modulate the oscilloscope beam intensity, so that each vertical line of the raster exhibits a varying brightness along the $y$ direction. Actually, a Schmidt descriminator placed after the photomultiplier is used to improve the intensity contrast, permitting, for each line, well defined dark and bright segments. These are shown in Fig. 3, which represents the scope pattern that can be recorded by a Polaroid camera. The dark and bright segments, which help to determine the positions of maxima and minima of the fringes, move as a function of time vs the horizontal direction, according to the fluctuations of the interference pattern. The rms of these displacements can be easily evaluated for each fringe.

The theoretical curve and the experimental values of the rms of the fringes fluctuation are plotted vs $\bar{l}$ in Fig. 4 by normalizing at a given point.

\section{Conclusions}

We have performed an experiment that allows us to test the diffusionlike behavior for the refractive index correlation function generated by thermal fluctuations. These have been artificially produced, although the given approach can furnish a first approximation model for some outdoors situations. This is, for example, the case in which thermal fluctuations are generated at ground level in presence of a slow ascending stream.

A simple calculation permits us to obtain under general hypothesis the expression of the mean square value of the phase fluctuations of a laser beam as a function of time. This dependence has been experimentally tested and a good agreement with the $1 / \bar{t}$ law has been found.

The validity of the model utilized is limited by the presence of the coupling terms that describe the interaction between scalar and velocity field. The diffusive hypothesis implies a dependence of $\left\langle(\Delta \phi)^{2}\right\rangle$ on $x$ and $v$ only through the ratio $\bar{t}=x / v$. Our experimental results show that this is no longer valid when the wind velocity is increased beyond a certain critical value, that in our case turns out to be of the order of $25 \mathrm{~cm} / \mathrm{sec}$.

We thank D. Sette and L. Muzii for much useful discussion and many suggestions. The assistance of $\mathrm{E}$. Bezzi in the experimental part of this work is gratefully acknowledged.

\section{References}

1. L. A. Chernov, Wave Propagation in a Random Medium (McGraw-Hill Book Co., Inc., New York, 1961).

2. V. I. Tatarski, Wave Propagation in a Turbulent Medium (McGraw-Hill Book Co., Inc., New York, 1961).

3. See for example, H. Bremmer, in Proceedings of the Symposium on Quasi-Optics (Polytechnic Press, Brooklyn, New York, 1964); F. Beckman, Radio Sci. 69 D, 629 (1965); D. A. DeWolf, J. Opt. Soc. Amer. 55, 812 (1965); J. I. Davis, Appl. Opt. 5, 139 (1966); H. Hodara, Proc. IEEE 54, 368 (1966); D. L. Fried, J. Opt. Soc. Amer. 57, 169, 175 (1967).

4. See for example, R. B. Herrick and J. R. Meyer, Appl. Opt. 5, 981 (1966); D. H. Höhn, Appl. Opt. 5, 1427 (1966); P. Burlamacchi, A. Consortini, and L. Ronchi, Appl. Opt. 6, 1273 (1967); M. Bertolotti, M. Carnevale, L. Muzii, and D. Sette, in Proceedings of the LIII Meeting of the Societa Italiana di Fisica (Società Italiana di Fisica, Bologna 1967).

5. J. O. Hinze, Turbulence (McGraw-Hill Book Co., Inc., New York, 1959) and bibliography therein. 

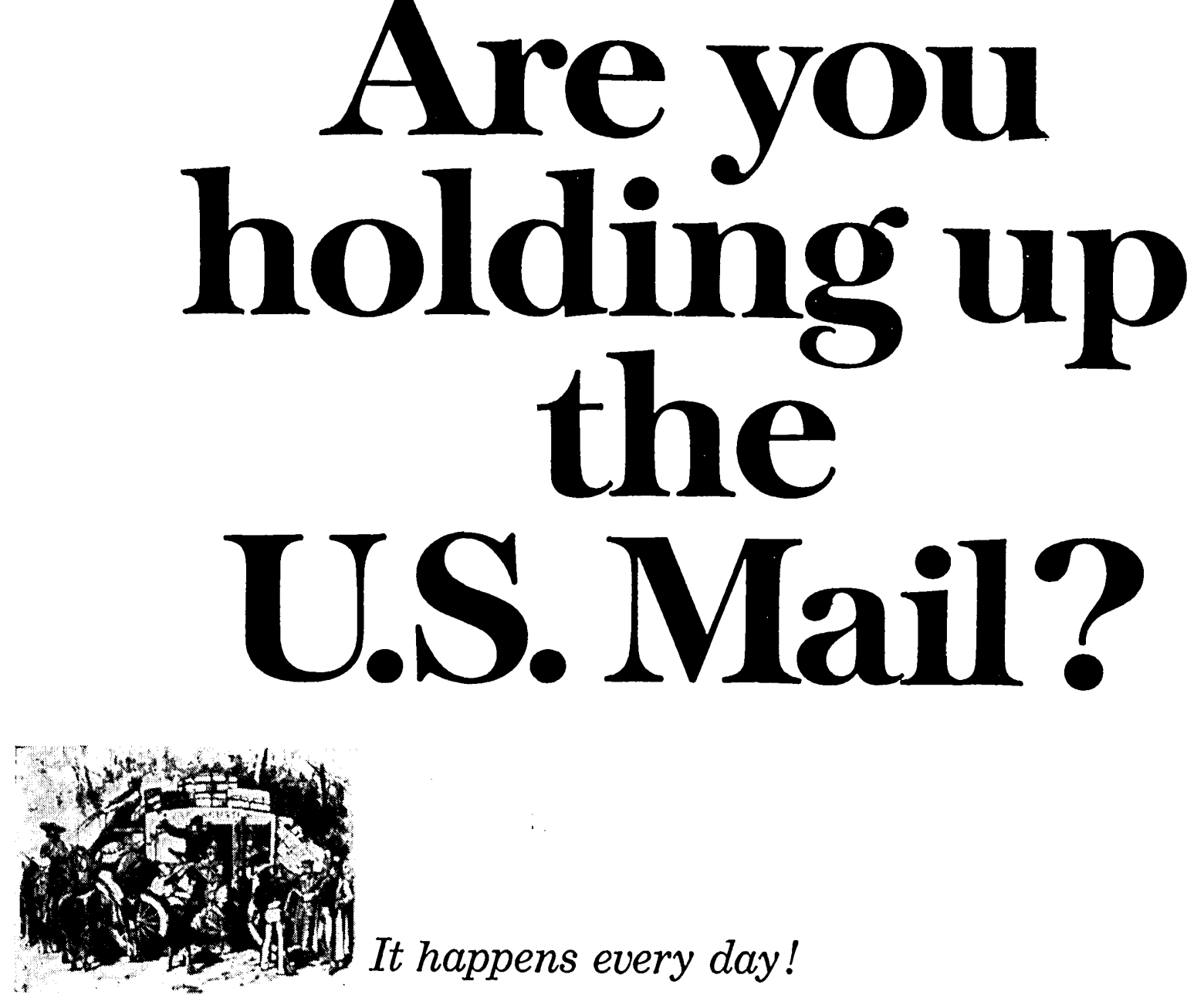

People hold up mail service for themselves and for everyone else by not using Zip Code on their mail.

Their mail has to be sorted and re-sorted several times along its route. A single unzipped letter can slow up the mail at 6 post offices!

But when your letter has Zip Code, postal workers can sort it faster and send it more directly to its destination.

They can also use modern electronic machines

that "read" Zip numbers and sort mail

fifteen times faster than was possible before.

So remember:

1. Always add Zip Code to every address you write to. If you don't know the number, call your post office or look it up in their Zip Code Directory. Local Zips can be found on the Zip Map in the business pages of your phone book.

2. Add Zip Code to your return address, too. That makes it easy for others to Zip their mail to you.

The post office must handle over 200 million new pieces of mail a day. Don't hold it up. Use Zip Code!

\section{Mail moves the country- ZIP CODE moves the mail!}

HOW ZIP CODE WORKS

Suppose the Zip Code is 60635. The " 6 " says it goes to the Midwest. The "06" narrows it down to Chicago. The last two digits-" 35 "-pinpoint the local post office. This eliminates many handling procedures. The letter is sorted faster, and sent more directly to its destination.

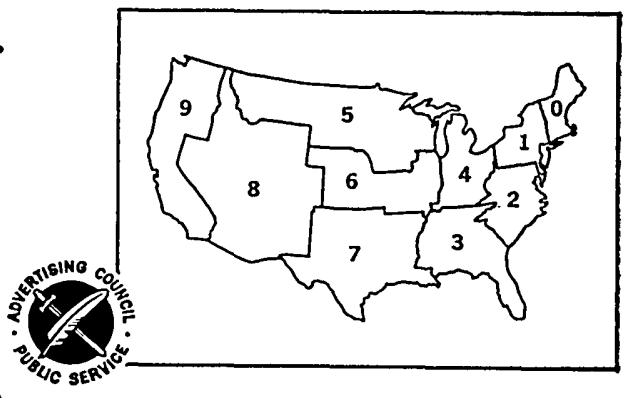

Published as a public service in cooperation with The Advertising Council 